\title{
Surface pinning in an amorphous ZrTiCuNiBe alloy
}

\author{
V. D. Fil, ${ }^{1}$ D. V. Fil,${ }^{2,3}$ Yu. A. Avramenko, ${ }^{1}$ A. L. Gaiduk, ${ }^{1}$ and W. L. Johnson ${ }^{4}$ \\ ${ }^{1}$ B. Verkin Institute for Low Temperature Physics and Engineering, National Academy of Sciences of Ukraine, Lenin av. 47, \\ Kharkov 61103, Ukraine \\ ${ }^{2}$ Institute for Single Crystals, National Academy of Sciences of Ukraine, Lenin av. 60, Kharkov 61001, Ukraine \\ ${ }^{3}$ Ukrainian State Academy of Railway Transport, Feyerbakh Sq. 7, Kharkov 61050, Ukraine \\ ${ }^{4}$ California Institute of Technology, Pasadena, California 91125, USA
}

(Received 23 December 2004; published 24 March 2005)

\begin{abstract}
We have measured the amplitude and the phase of an electromagnetic (EM) field radiated from a superconductor (amorphous ZrTiCuNiBe alloy) in the mixed state due to the interaction of the flux lattice with an elastic wave. The results point to an essential contribution of a surface pinning into the flux lattice dynamics. We propose a model that describes radiation of the EM field from superconductors with nonuniform pinning. The model allows us to reconstruct the viscosity and the Labusch parameters from the experimental data. The behavior of the Labusch parameter can be qualitatively explained in terms of the collective pinning theory with the allowance of thermal fluctuations.
\end{abstract}

DOI: 10.1103/PhysRevB.71.092504

PACS number(s): 74.25.Qt, 74.70.Ad

Soft type-II superconductors were studied intensively during last four decades with the goal to investigate various aspects of vortex matter dynamics (see the comprehensive review by $B^{2} \mathrm{dt}^{1}$ ). Nevertheless, many questions that require further investigation remain. Among these questions is the problem of relation between the surface and the bulk pinning. Up to now the dynamics of the vortex state in such an exclusively nonuniform situation was studied by the surface impedance method. ${ }^{2}$ In this paper, on the example of amorphous $\mathrm{Zr}_{41.2} \mathrm{Ti}_{13.8} \mathrm{Cu}_{12.5} \mathrm{Ni}_{10} \mathrm{Be}_{22.5}$ alloy we demonstrate abilities of a method based on excitation of vortex lattice oscillations by a high-frequency sound wave.

The essence of the method is the following. A superconductor situated in the lower half space $(z<0)$ is subjected by a constant magnetic field $\mathbf{H} \| z$. A transverse elastic wave propagating along $\mathbf{H}$ and polarized in $x$ direction produces transverse (with respect to $\mathbf{H}$ ) oscillations of the vortex lattice caused by pinning and viscous friction, and, consequently, induces an electromagnetic (EM) field. An antenna receives the EM field (with $E_{y}$ and $H_{x}$ components) radiated through the elastically free surface of the sample (the surface perpendicular to the direction of propagation of the elastic wave). While a similar experimental setup was already applied for the study of type-II superconductors, ${ }^{3,4}$ the key point is measuring both the amplitude and the phase of the EM field (more accurately, the changes of these quantities).

In the uniform case and in the local limit $\left(q \ll l^{-1}, q\right.$ is the wave number and $l$ is the mean-free path) the components of the EM field at $z=0$ are given by a simple expression, 5,6

$$
H_{x}=E_{y}=\frac{\dot{u}(0)}{c} H \frac{k^{2}}{q^{2}+k^{2}},
$$

where $u(z)=u_{0} \cos q z e^{i \omega t}$ is the displacement in the elastic wave, $k^{2}$ is the square of the complex wave number of the EM field in a conductor, and $c$ is the light velocity.

Equation (1) is applicable for the normal as well as the superconducting state of the metal. In the normal state $k^{2}$ $=k_{n}^{2}=4 \pi i \omega \sigma_{0} / c^{2}\left(\sigma_{0}\right.$ is the static conductivity in the normal state) is imaginary valued quantity. In the superconducting state at small magnetic fields $\left(H \sim H_{c 1}\right) k^{2}=k_{s}^{2}=\lambda_{L}^{-2}\left(\lambda_{L}\right.$ is the London penetration length) is real valued. At such fields the phase of the EM signal $\varphi$ is a constant and we use its value as the reference point. In the well-developed Shubnikov state $k^{2}=k_{m}^{2}=4 \pi\left(i \omega \eta+\alpha_{L}\right) / H^{2}$, where $\eta>0$ is the viscosity parameter and $\alpha_{L}>0$ is the Labusch "spring" parameter. As follows from Eq. (1), the phase is positive at all $H>H_{c 1}$ and it approaches $\varphi_{n}<90^{\circ}$ (the phase in the normal state) at $H$ $\rightarrow H_{c 2}$.

The initial goal of our experiment was to obtain experimentally the dependencies $\eta(H)$ and $\alpha_{L}(H)$ using Eq. (1), as it was done before for $\mathrm{MgB}_{2} \cdot{ }^{7}$ We use the working frequency $\omega / 2 \pi \sim 55 \mathrm{MGz}$. Details of the measuring procedure are described in Ref. 8. The results of our first experiments are presented in Fig. 1.

Prior to discussing these results let us give the values of parameters for the material under study. The dc resistivity $\rho \approx 200 \mu \Omega \mathrm{cm}$ is practically temperature independent. The Alfer-Rubin effect $^{9}$ (the dependence of the sound attenuation coefficient on the magnetic field) allows us to determine the parameter $\beta=\left|q^{2} / k_{n}^{2}\right|=91 \pm 1$, in excellent coincidence with the value of $\rho$ measured $\left(q=1400 \mathrm{~cm}^{-1}\right.$, see Ref. 10). The

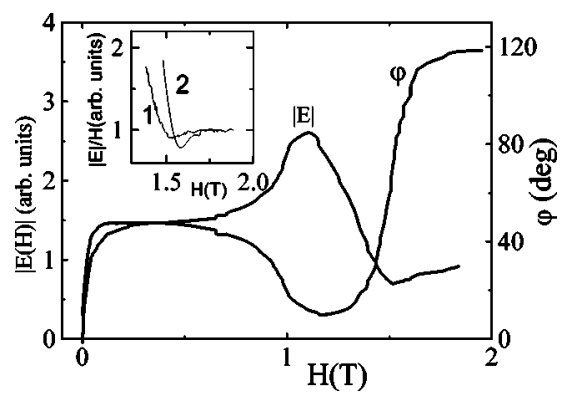

FIG. 1. Amplitude and phase of the EM field measured for the sample with imperfect surface. Inset-behavior of $|E| / H$ near $H_{c 2}$ [1-experiment, 2 - computed from Eq. (7) with $\alpha_{L}^{\text {eff }}$ (see below)]. 
Hall constant, measured at room temperature, is positive and very small: $R_{H}=1 /$ enc $=(3.2 \pm 0.4) \times 10^{-25}$ cgs units, that yields a rather high density of the carriers $n \approx 2.2 \times 10^{23}$ $\mathrm{cm}^{-3}$. Taking $m=m_{e}$, we obtain the relaxation time $\tau \approx 0.8$ $\times 10^{-16} \mathrm{~s}$, and for the Fermi velocity $v_{F} \sim 10^{8} \mathrm{~cm} / \mathrm{s}$ we find $l \sim 10^{-8} \mathrm{~cm}$. It was shown before ${ }^{11}$ that the alloy under study belongs to the family of weak-coupling superconductors with the standard BCS energy gap $\Delta(0) \approx 1.75 T_{c}\left(T_{c} \approx 0.85 \mathrm{~K}\right)$. In the dirty limit the formula for the penetration depth ${ }^{12}$ can be rewritten as $\lambda_{L}^{-2}=k_{n}^{2}(\Delta / i \omega) \tanh (\Delta / 2 T)$, that yields $\lambda_{L}=3$ $\times 10^{-4} \mathrm{~cm}$ at $T=0.4 \mathrm{~K}$. The coherence length, calculated from $H_{c 2}$, is $\xi(0.4 \mathrm{~K})=1.4 \times 10^{-6} \mathrm{~cm}$.

As follows from Eq. (1), for $\beta$ given above one could expect $\varphi_{n} \approx 90^{\circ}$ that contradicts the results presented in Fig. $1\left(\varphi_{n} \approx 120^{\circ}\right)$. Let us show that this discrepancy can be accounted for a lowering of the conductivity near the surface of the sample caused by defects.

We imply the following model dependence of the conductivity on $z: \sigma_{0}(z)=\sigma_{0 v}\left[1-p \exp \left(z / z_{\sigma}\right)\right]$ (with $p \leqslant 1$ and $z_{\sigma}$ $>0$ ). Then, in the normal state, the electrodynamic equation for the EM field has the form ${ }^{6}$ [compare with Eq. (4) below]

$$
\frac{d^{2} \widetilde{E}}{d \zeta^{2}}-a(\zeta) \widetilde{E}=a(\zeta) \cos (\zeta)
$$

where $a(\zeta)=a\left[1-p \exp \left(\zeta / \zeta_{0}\right)\right], a=k_{n v}^{2} / q^{2}, k_{n v}^{2}=4 \pi i \omega \sigma_{0 v} / c^{2}$, $\zeta_{0}=q z_{\sigma}$. Here we use the dimensionless variables $\tilde{E}$ $=E c /\left(i \omega u_{0} H\right)$ and $\zeta=q z$. The boundary conditions for Eq. (2) are that $|\widetilde{E}(\zeta)|$ is finite at all $\zeta$ and $d \tilde{E} /\left.d \zeta\right|_{\zeta=0}=0$. The latter condition is due to continuity of the EM field on the conductor-vacuum interface and is valid with the accuracy $\delta / \lambda_{E M} \lesssim 10^{-4}$ ( $\delta$ is the skin depth and $\lambda_{E M}$ is the wavelength of the EM field in vacuum).

The solution of Eq. (2) can be expressed through the Bessel functions of the first kind of complex order $\nu$ $=2 \zeta_{0} \sqrt{a}$ on the complex variable $t(\zeta)=2 \zeta_{0} \sqrt{p a} \exp \left(\zeta / 2 \zeta_{0}\right)$. The field at the conductor-vacuum interface is

$$
\widetilde{E}(z=0)=\left(\left.\frac{d J_{\nu}[t(\zeta)]}{d \zeta}\right|_{\zeta=0}\right)^{-1} \int_{-\infty}^{0} d \zeta J_{\nu}[t(\zeta)] a(\zeta) \cos \zeta .
$$

One can evaluate Eq. (3) using the expansion of $J_{\nu}(t)$ in series in $t$ and integrating each term analytically. For the case of interest it is enough to take into account the first two terms of the expansion.

Analysis of Eq. (3) shows that the phase $\varphi_{n}$ increases under increasing $p$. In our case of the extremely dirty conductor and for $\zeta_{0} \sim 1\left(z_{\sigma} \approx 7 \mu \mathrm{m}\right)$ we find that a rather small lowering of the surface conductivity $(p \sim 0.1)$ results in increasing of $\varphi_{n}$ up to $120^{\circ}$.

Removing the layer $\sim 50 \mu \mathrm{m}$ from the surface of the sample by additional polishing with a fine powder we managed to eliminate the effect caused by the lowering of surface conductivity. The results of measurements (at various $T$ ) are shown in Fig. 2. One can see that in this case $\varphi_{n} \approx 90^{\circ}$.

The main peculiarity of the dependencies presented in Fig. 2 is that the phase $\varphi$ becomes negative at intermediate

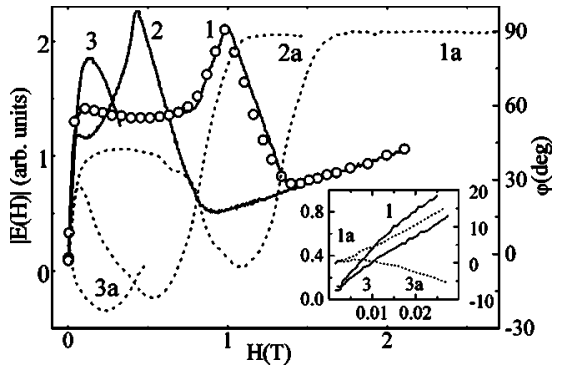

FIG. 2. Amplitude (solid curves 1, 2, 3) and phase (dotted curves $1 \mathrm{a}, 2 \mathrm{a}, 3 \mathrm{a}$ ) of the EM field measured for the sample with a perfect surface at $T=0.4,0.69,0.8 \mathrm{~K}$, respectively (for $T=0.8 \mathrm{~K}$ the results for the normal state are not shown). Circles- the dependence $|E(H)|$ measured under decreasing of $H$. Inset-the same dependencies in the interval of small magnetic fields (all notations are the same as in the main plot).

magnetic fields. This effect cannot be described by Eq. (1) under any reasonable variation of $\eta$ and $\alpha_{L}$ with $H$.

In what follows we will argue that the effect observed can be explained by nonuniform pinning near the surface of the sample that takes place even for uniform $\sigma_{0}$ (and, consequently, for uniform $\eta$ ).

The EM field in the mixed state is described by the system of equations, namely, the Maxwell equation, the matter equation, that determines the value of the current, and the equation of motion for the vortex lattice, ${ }^{6}$

$$
\begin{gathered}
\frac{d^{2} \mathbf{E}}{d z^{2}}=\frac{4 \pi i \omega}{c^{2}} \mathbf{j}=k_{s}^{2}\left(\mathbf{E}+\frac{1}{c} \dot{\mathbf{u}}_{v} \times \mathbf{H}\right), \\
\frac{1}{c} \mathbf{j} \times \mathbf{H}+i \omega \eta\left(\mathbf{u}-\mathbf{u}_{v}\right)+\alpha_{L}\left(\mathbf{u}-\mathbf{u}_{v}\right)=0,
\end{gathered}
$$

where $\mathbf{u}_{v}$ is the displacement of the vortex lattice. Here we neglect the normal component of the current.

Nonuniform pinning can be modeled by the $z$-dependent Labusch parameter. We specify this dependence as $\alpha_{L}(z)$ $=\alpha_{L v}+\alpha_{L s} \exp \left(z / z_{p}\right)$. Using Eqs. (4) and (5) we obtain the equation for the EM field in the mixed state. At the magnetic field that satisfies the inequality $k_{s}^{2}\left(H^{2} / 4 \pi\right) \gg\left|i \omega \eta+\alpha_{L}\right|$, this equation coincides in form with Eq. (2) with the same boundary conditions and the quantity $a(\zeta)$ defined as

$$
a(\zeta)=\frac{i \omega \eta+\alpha_{L v}}{q^{2} \frac{H^{2}}{4 \pi}}+\frac{\alpha_{L s}}{q^{2} \frac{H^{2}}{4 \pi}} \exp \frac{\zeta}{\zeta_{0}} \equiv A+C \exp \frac{\zeta}{\zeta_{0}},
$$

where $\zeta_{0}=q z_{p}$.

Taking into account the first two terms in the expansion of the Bessel function we obtain from Eq. (3) the approximate expression 


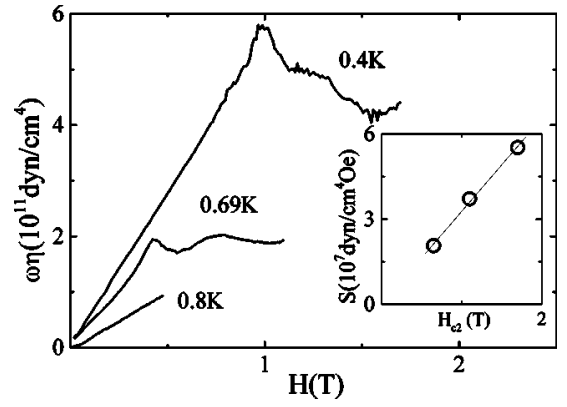

FIG. 3. Values of $\omega \eta$ reconstructed from the data in Fig. 2 using Eq. (7). Inset-the slope $S$ of $\omega \eta$ for intervals of linear increase of $\omega \eta$ with $H$ (circles); solid line-linear fit.

$$
\tilde{E}(0)=\frac{\sqrt{A} \frac{A}{1+A}+C \frac{\zeta_{0}}{1+\zeta_{0}^{2}}}{\sqrt{A}+C \zeta_{0}}
$$

In contrast to Eq. (1), that requires $\eta<0$ ("negative viscosity") to describe negative phases of the EM signal, Eq. (7) may yield negative $\varphi$ at positive $\eta$.

Equation (7) can be used to reconstruct the dependencies $\eta(H)$ and $\alpha_{L}(H)$ from the experimental data presented in Fig. 2 , if the parameters $\zeta_{0}$ and $\alpha_{L v} / \alpha_{L s}$ are specified. These parameters are not known, but there are limitations on the choice of them. We require the functions $\eta(H)$ and $\alpha_{L}(H)$ to be smooth and real valued. Numerical analysis shows that these requirements satisfy for $\alpha_{L v} \ll \alpha_{L s}$ (it means that the bulk pinning can be neglected) and $\zeta_{0} \lesssim 0.5$. At such conditions the amplitude and the phase of the EM signal are, in fact, determined by two parameters: $\eta$ and $\zeta_{0} \alpha_{L s}$, and the procedure of reconstruction of $\eta(H)$ and $\zeta_{0} \alpha_{L S}(H)$ can be done without any additional assumption.

The dependencies $\eta(H)$ are shown in Fig. 3. In a rather wide interval of $H$ these dependencies are linear with the slope $S \propto H_{c 2}$ (Fig. 3, inset) and are described by the formula $\eta(H)=(2 \pm 0.05) H_{c 2} \sigma_{0} / c^{2}$. Such type of dependencies are typical for the mixed state and we consider it as a confirmation of the model proposed.

The dependencies $\zeta_{0} \alpha_{L s}(H)$ are shown in Fig. 4. They

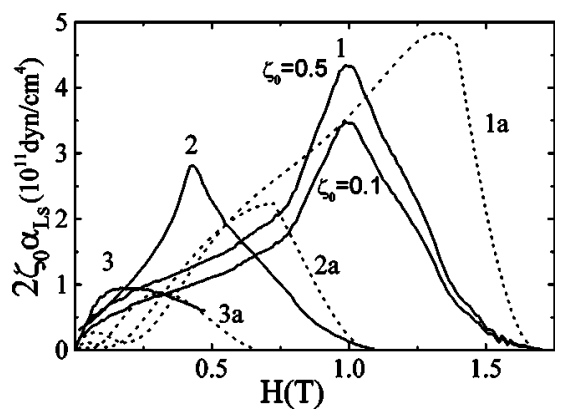

FIG. 4. Solid curves-values of $\zeta_{0} \alpha_{L s}$ reconstructed from the data in Fig. 2 using Eq. (7) at $\zeta_{0}=0.5$ and $\zeta_{0}=0.1$ (only for $T$ $=0.4 \mathrm{~K})$; dashed curves - computed dependence $\alpha_{L}^{\text {eff }}(H)$ at $\zeta_{0}=0.5$ (see text). 1 and $1 \mathrm{a}$ curves are for $T=0.4 \mathrm{~K} ; 2$ and $2 \mathrm{a}$ for $T$ $=0.69 \mathrm{~K}$; and 3 and $3 \mathrm{a}$ for $T=0.8 \mathrm{~K}$. demonstrate two peculiarities: the strong asymmetry of the function $\alpha_{L S}(H)$ at $T=0.4 \mathrm{~K}$ and a rather slow decreasing of $\alpha_{L s}$ under lowering of $H_{c 2}$ (usually $\alpha_{L} \propto H_{c 2}^{n}$, where $n=2-3$ ). We will show below that such a behavior can be explained at least semiquantitatively in the framework of the accepted theories of the vortex state dynamics.

Down to the lowest temperatures available in our experiment we did not find (see Fig. 2) any signs of freezing of the magnetic flux (the irreversibility line). It means that the pinning is quite weak and it can be described by the collective pinning $(\mathrm{CP})$ theory. ${ }^{13}$ The $\mathrm{CP}$ theory contains an additional parameter connected with quenched disorder of the vortex lattice caused by a random pinning potential. It is convenient to choose a field $H_{s v} \leqslant 0.5 H_{c 2}$ as such a parameter. ${ }^{14}$ This field separates the regime of single vortex pinning (SVP) from the regime of bundle vortex pinning (BVP). In the SVP regime $\left(H \leqslant H_{s v}\right.$ or $\left.H \geqslant H_{c 2}-H_{s v}\right)$ the value of $\alpha_{L}$ is given by the expression $\alpha_{L} \approx C_{66} a_{v}^{-2}\left(H_{s v}\right)$, where $C_{66}$ $=\left[\Phi_{0} H /\left(8 \pi \lambda_{L}\right)^{2}\right]\left[1-\left(H / H_{c 2}\right)\right]^{2}$ is the shear modulus of the vortex lattice, $a_{v}(H)=\sqrt{\Phi_{0} / H}$ is the vortex lattice constant, and $\Phi_{0}$ is the quantum of magnetic flux. In the BVP regime $\left(H_{s v} \leqslant H \leqslant H_{c 2}-H_{s v}\right)$ the Labusch parameter is $\alpha_{L}$ $\approx C_{66} R_{c}^{-2}(H)$, where the collective pinning radius $R_{c}$ is given by the formula ${ }^{15}$

$$
R_{c}(H) \approx a_{v}(H) \exp \left\{\frac{1}{2}\left[\left(\frac{H\left(H_{c 2}-H\right)}{H_{s v}\left(H_{c 2}-H_{s v}\right)}\right)^{3 / 2}-1\right]\right\} .
$$

We note that Eq. (8) determines the dependence of $\alpha_{L}(H)$ almost symmetric relative to the $H=0.5 H_{c 2}$ axis.

One can see that the scale of $\alpha_{L}$ is determined mainly by the value of $C_{66}$. But one finds from the data in Fig. 4 that even for maximum $\zeta_{0}\left(\zeta_{0}=0.5\right.$, and $\left.z_{p}=3.5 \mu \mathrm{m}\right)$ the scale of measured $\alpha_{L}$ exceeds significantly the estimate that follows from the value of $\lambda_{L}$ given above. Probably, it is connected with the fact that the relation for $\lambda_{L}$ overestimates this quantity. Unfortunately, in our experiment we cannot measure the field $H_{c 1}$ directly with an accuracy sufficient to refine the value of $\lambda_{L}$. Assuming $\zeta_{0}=0.5$, we model $\alpha_{L}(H)$, taking $C_{66}$ in six times larger than it follows from the estimate of Ref. 12 and implying their standard temperature dependence [that corresponds to $\left.\lambda_{L}(0.4 \mathrm{~K})=1.2 \times 10^{-4} \mathrm{~cm}\right]$.

Since the system is in a weak pinning regime we must take into account temperature fluctuations. To evaluate the effect of thermal fluctuations one should add a random force $f_{L}(T)$ into Eq. (5). ${ }^{16}$ Strictly speaking, the returning force $j H / c=\left(H^{2} / 4 \pi\right) \partial^{2} u_{v} / \partial z^{2}$ in Eq. (5) has to be found from a joint solution of the system (4) and (5), but for the estimates one can replace $\partial^{2} / \partial z^{2}$ with $-q^{2}$. In this case Eq. (5) can be rewritten in the form of an equation of diffusion of the Brownian particle

$$
\tilde{\eta} \frac{\partial w}{\partial t}=-\frac{\partial}{\partial w}\left(\tilde{V}_{0}+\tilde{V}_{u}+\tilde{V}_{p}\right)+f_{L}(T),
$$

where $w=u-u_{v}$, the "tildes" indicate that the corresponding quantities are given per one diffusing "particle:" $\tilde{y}$ $=y \Phi_{0} L_{c} / H \quad\left(L_{c}\right.$ is the collective pinning length $), V_{0}$ $=\left(q^{2} H^{2} / 8 \pi\right) w^{2}, V_{u}=-\left(q^{2} H^{2} / 4 \pi\right) u w$, and $V_{p}$ is the pinning potential. Taking into account equal probabilities of diffuse 
motion of a vortex in opposite directions we model $V_{p}$ by a three-well potential

$$
V_{p}=\frac{\alpha_{L}}{2} \begin{cases}(w+d)^{2} & w \leqslant-d / 2 \\ w^{2} & |w| \leqslant d / 2 \\ (w-d)^{2} & w \geqslant d / 2\end{cases}
$$

where $d$ is the distance between the minima of the pinning potential. The linearized Fokker-Planck equation that corresponds to Eq. (9) with the pinning potential (10) has the exact solution in terms of hypergeometric functions. At $\alpha_{L} /\left[q^{2}\left(H^{2} / 4 \pi\right)\right] \ll 1$ the following simple estimate for the averaged displacement of the vortex lattice is found $\left\langle u_{v}\right\rangle$ $\approx u \cdot 4 \pi\left(i \omega \eta+\alpha_{L}^{e f f}\right) /\left(q^{2} H^{2}\right)$, where $\alpha_{L}^{e f f}=\alpha_{L}\left[1-(4 / \sqrt{\pi}) c e^{-c^{2}}\right]$ and $c^{2}=\left(q^{2} H \Phi_{0} L_{c} / 2 \pi T\right) d^{2}$. One can see from the comparison of this formula with Eq. (5) that the thermal fluctuations reduce the effective Labusch parameter, but the viscosity parameter remains unchanged.

For the general case the dependencies $\alpha_{L}^{e f f}(H)$ obtained from the solution of the Fokker-Planck equation are shown in Fig. 4. We use the following parameters for the computations. To achieve semiquantitative agreement between the theoretical results and the experimental data at $T=0.4 \mathrm{~K}$ we set $H_{s v}(0.4) \approx 0.15 H_{c 2}(0.4)$ and $d(0.4) \approx 5 \times 10^{-7} \mathrm{~cm}$ (as expected, $d$ is of order of $\xi$ ). We assume that the temperature dependence of $H_{s v}$ is determined by the $\delta T_{c}$ pinning: $H_{s v} / H_{c 2} \propto\left[1-\left(T / T_{c}\right)^{2}\right]^{-1 / 3}$. $^{14}$ It looks quite reasonable, because there are inclusions of another superconducting phase with higher $T_{c}$ on the surface of our sample. ${ }^{11}$ The temperature dependence $d(T) \propto \xi(T)$ is implied. We set $L_{c}=a_{v}(H)$.

One can see from Fig. 4 that the computed temperature and field dependencies of $\alpha_{L}$ are in qualitative agreement with the experimental results.

It is interesting to note that if we substitute $\alpha_{L}^{\text {eff }}$ into Eq. (7) we obtain $|E| / H$ that has a local minimum near $H_{c 2}$. Such a minimum is observed sometimes in our experiment (Fig. 1, inset) as well as in Ref. 3. Physically this minimum, as well as negative $\varphi$, is caused by that the surface layer with a reduced penetration depth screens the EM field radiated from deeper regions of the sample. Reminiscence of this minimum is also present in Fig. 2: under transition from the normal to mixed state the increase of $|E|$ begins only after a substantial lowering of $\varphi$.

In conclusion, we propose a method of investigating the dynamical characteristics of the vortex matter that consists in measuring the amplitude and the phase of the EM field radiated from the sample under excitation of the vortex lattice oscillations by the elastic wave. It is established that unusual behavior of the amplitude and the phase of the EM field are accounted for the nonuniform pinning. The parameters measured (the viscosity coefficient and the Labusch parameter) are in qualitative agreement with the estimates obtained from the accepted theories of vortex matter dynamics.

This study was supported in part by CRDF (Grant No. UP1-2566-KH-03), by INTAS (Grant No. 03-51-3036), and by Ukrainian Government Foundation for Basic Research (Grant No. 0207/00359). We would like to thank G. P. Mikitik and K. V. Maslov for helpful discussions.
${ }^{1}$ E. H. Brandt, Rep. Prog. Phys. 58, 1465 (1995).

${ }^{2}$ N. Lütke-Eutrup, B. Placais, P. Mathien, and Y. Simon, Physica B 255, 75 (1998).

${ }^{3}$ L. A. Vienneau and B. W. Maxfield, Phys. Rev. B 11, 4339 (1975).

${ }^{4}$ H. Haneda and T. Ishiguvo, Physica C 235-240, 2076 (1994).

${ }^{5}$ D. Dominguez, L. Bulaevskii, B. Ivlev, M. Maley, and A. R. Bishop, Phys. Rev. B 51, 15649 (1995).

${ }^{6}$ V. D. Fil, Low Temp. Phys. 27, 993 (2001).

${ }^{7}$ T. V. Ignatova, G. A. Zvyagina, I. G. Kolobov, E. A. Masalitin, V. D. Fil, Yu. B. Paderno, A. N. Bykov, and V. I. Lyashenko, Low Temp. Phys. 28, 190 (2002).

${ }^{8}$ E. A. Masalitin, V. D. Fil, K. R. Zhekov, A. N. Zholobenko, T. V. Ignatova, and S.-I. Lee, Low Temp. Phys. 29, 72 (2003).
${ }^{9}$ R. A. Alfer and R. J. Rubin, J. Acoust. Soc. Am. 26, 452 (1954).

${ }^{10}$ A. L. Gaiduk, E. V. Bezuglyi, V. D. Fil, and W. L. Johnson, Low Temp. Phys. 23, 857 (1997).

${ }^{11}$ E. V. Bezuglyi, A. L. Gaiduk, V. D. Fil, S. Zherlitsyn, W. L. Johnson, G. Bruls, B. Lüthi, and B. Wolf, Phys. Rev. B 62, 6656 (2000).

${ }^{12}$ A. A. Abrikosov, Fundamentals of the Theory of Metals (NorthHolland, Amsterdam, 1998).

${ }^{13}$ G. Blatter, M. V. Feigel'man, V. B. Geshkenbein, A. I. Larkin, and V. M. Vinokur, Rev. Mod. Phys. 66, 1125 (1994).

${ }^{14}$ G. P. Mikitik and E. H. Brandt, Phys. Rev. B 64, 184514 (2001).

${ }^{15}$ G. P. Mikitik (private communication). Equation (8) is obtained from Eqs. (4.17) and (4.18) of Ref. 13, and Eq. (8) of Ref. 14. ${ }^{16}$ M. W. Coffey and J. R. Clem, Phys. Rev. B 45, 9872 (1992). 